\title{
Men with chronic prostatitis at risk for colorectal cancer?
}

A case-control study from Taiwan, published online in BJU International, has reported an intriguing association between chronic prostatitis/chronic pelvic pain syndrome (CP/CPPS) and colorectal cancer.

Investigators retrospectively analyzed data from the Longitudinal Health Insurance Database 2000, a large repository of information on the insurance claims of 1 million beneficiaries of Taiwan's National Health Insurance scheme. They identified 2,899 men over 40 years old who were diagnosed with colorectal cancer between 2002 and 2008, and matched each individual with five control patients who had never been diagnosed with any type of cancer.

The study found that $4.24 \%$ of men with colorectal cancer had previously been diagnosed with CP/CPPS, compared with only $2.81 \%$ of patients in the control group. After adjusting for a number of sociodemographic and medical characteristics, this translated to a 1.45 -fold increased risk of previous
$\mathrm{CP} / \mathrm{CPPS}$ in men with colorectal cancer (95\% CI 1.17-1.79; $P<0.001$ ). Subgroup analysis revealed that this relationship between colorectal cancer and CP/CPPS was limited to patients under the age of 60 years. The strongest effect was noted in

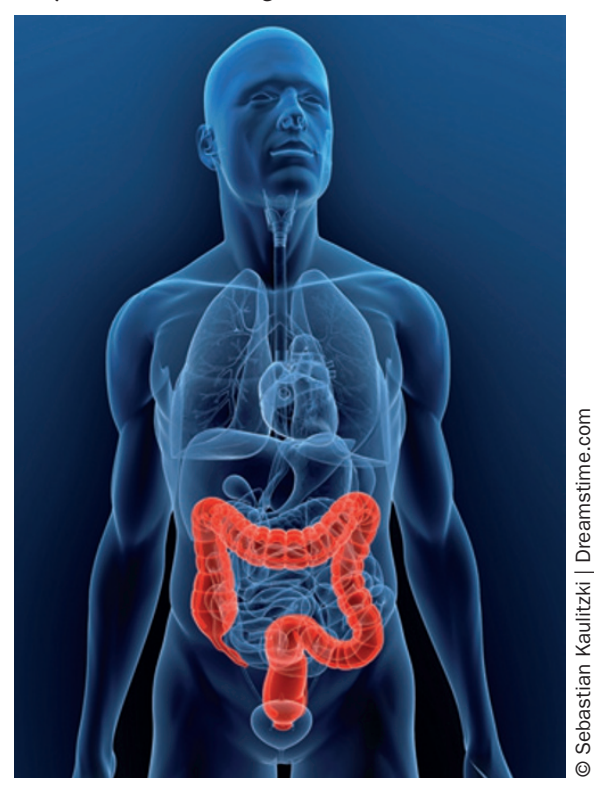

men aged 50-59 years (OR 2.40; 95\% CI

1.49-3.87; $P<0.001)$.

Given the well-established link between intestinal inflammation and development of colorectal cancer, the authors hypothesize that inflammation in the prostate might also be anatomically relevant. Although this represents an interesting future avenue of research, there are some major caveats to keep in mind with regards to this study. Using claims data for disease diagnosis has inherent problems, which might be exacerbated in the case of CP/CPPS-a poorly understood disorder with no definitive diagnostic tests. It is especially noteworthy that the single classification code used to define a diagnosis of CP/CPPS in this study covers a broad range of prostatitis-like symptoms.

\section{Sarah Payton}

Original article Chung, S. D. et al. A case-control study of chronic prostatitis/chronic pelvic pain syndrome and colorectal cancer. BJU Int. doi:10.1111/j.1464410X.2012.10929.x. 